\title{
Antinociceptive tolerance to NSAIDs in the anterior cingulate cortex is mediated via endogenous opioid mechanism
}

\author{
Nana Tsiklauri, Natia Pirkulashvili, Ivliane Nozadze, Marina Nebieridze, Gulnaz Gurtskaia, Elene Abzianidze \\ and Merab G. Tsagareli ${ }^{*}$
}

\begin{abstract}
Background: In the past decade several studies have reported that in some brain areas, particularly, in the midbrain periaqueductal gray matter, rostral ventro-medial medulla, central nucleus of amygdala, nucleus raphe magnus, and dorsal hippocampus, microinjections of non-steroidal anti-inflammatory drugs (NSAIDs) induce antinociception with distinct development of tolerance. Given this evidence, in this study we investigated the development of tolerance to the analgesic effects of NSAIDs diclofenac, ketorolac and xefocam microinjected into the rostral part of anterior cingulate cortex (ACC) in rats.
\end{abstract}

Methods: Male Wistar experimental and control (saline) rats were implanted with a guide cannula in the ACC and tested for antinociception following microinjection of NSAIDs into the ACC in the tail-flick (TF) and hot plate (HP) tests. Repeated measures of analysis of variance with post-hoc Tukey-Kramer multiple comparison tests were used for statistical evaluations.

Results: Treatment with each NSAID significantly enhanced the TF and HP latencies on the first day, followed by a progressive decrease in the analgesic effect over a 4-day period, i.e., developed tolerance. Pretreatment with an opioid antagonist naloxone completely prevented the analgesic effects of the three NSAIDs in both behavioral assays.

Conclusions: These findings support the concept that the development of tolerance to the antinociceptive effects of NSAIDs is mediated via an endogenous opioid system possibly involving descending pain modulatory systems.

Keywords: Antinociception, Endogenous opioids, Descending modulation, Nociception, Non-opioid tolerance

\section{Background}

The most important signaling mechanism for imminent harm is the pain system. Studies of the emotional and motivational basis of pain reveal a diverse and complex set of processes by which the affective experience of pain is realized. In particular, the perception of both pain intensity and aversiveness is the complex process by which the brain constructs the sensory and emotional sensation of pain and challenges any standard "perception-action" model [1].

The first anatomical, physiological and behavioral investigations have demonstrated the important role of the

\footnotetext{
* Correspondence: m.tsagareli@biomedicine.org.ge; merab.tsagareli@caucasus.net

Lab of Pain and Analgesia, Beritashvili Center for Experimental Biomedicine, 14, Gotua Street, 0160 Tbilisi, Georgia
}

brain limbic system in the affective-motivational component of pain. Some clinical evidence and animal studies have shown the importance of the anterior cingulate cortex (ACC) in affective aspects of pain [2]. Injection of local anesthetics into the lateral hypothalamus, the cingulum bundle and the dentate nucleus of the hippocampus has temporarily blocked neural activity and induced significant analgesia during late tonic phase of pain perception [3-5]. Furthermore, surgical lesions of the cingulate cortex and/or the cingulum bundle were described as able to reduce the emotional but not the sensory component of chronic pain [6]. On the strength of positron emission tomography scan images, it has been concluded that activation of structures associated with autonomic and limbic system functions, such as the 
insula and the ACC, may reflect the affective aspect of pain experience [7].

Nowadays, it is well known that the ACC is involved in pain perception primarily receiving extensive projections from the medio-dorsal thalamic nucleus and broadly connects with relevant regions of the descending pain modulation system. A projection from the spinal dorsal horn through the medial/intra-laminar thalamic nuclei to the ACC has been proposed to process information on pain-related unpleasantness. In addition to contributing to the immediate affective consequences of noxious stimulation, the 'ACC system' may contribute to the avoidance learning that sometimes follows as a secondary reaction to pain. Neurons in the rostral ACC are required for painrelated aversion learning - a process that directly reflects the affective component of pain [8]. Just recently, in chronic pain patients proton magnetic resonance spectroscopy has revealed a different metabolite status in the AAC compared to controls. The mean levels of glutamic acid (Glu)/total creatine and Glu + glutamine/total creatine are higher, but lower for $\mathrm{N}$-acetylaspartate/total creatine, compared with healthy controls [9].

Unlike opioid analgesics, non-steroidal anti-inflammatory drugs (NSAIDs) are the most widely used analgesics in the treatment of mild, not severe pain. For the last years, however, it was established that repeated administration of NSAIDs systemically or in the midbrain periaqueductal grey matter (PAG) induced tolerance to these drugs, similarly to opioid analgesics, and cross-tolerance to morphine [10-16].

We have recently shown that tolerance develops to analgesic effects of the commonly used NSAIDs metamizol, diclofenac, ketorolac and xefocam given intraperitoneally (i.p.) in juvenile and adult rats in models of acute pain $[17,18]$. We have also revealed that repeated microinjections of these non-opioids into the dorsal hippocampus (DH), the nucleus raphe magnus (NRM), and the central nucleus of amygdala (CeA), induce antinociception and the effects of tolerance and crosstolerance to morphine [13,14, 19-21]. These findings strongly support the suggestion of endogenous opioids involvement in NSAIDs antinociception and tolerance in the descending pain-control system [13, 22].

In the present study, we hypothesized that the analgesic effects of the three NSAIDs, diclofenac, ketorolac and xefocam microinjected into the ACC would exhibit tolerance mediated via endogenous opioids.

\section{Methods}

\section{Animals}

The experiments were performed on male Wistar rats weighing 200 to $250 \mathrm{~g}$ at the time of surgery bred at the Beritashvili Exp. BMC. The animals were kept under standard housing conditions $\left(22 \pm 2{ }^{\circ} \mathrm{C}, 65 \%\right.$ humidity, and light from 7:00 a.m. to 8:00 p.m.) and kept on a standard dry diet with water freely available. Every procedure was designed to minimize discomfort to animals and efforts were made to minimize their number. Six rats were used for each experimental and control groups. All procedures adhered to the Guidelines of the International Association for the Study of Pain regarding animal experimentation [23] and were approved by Institutional Animal Care and Use Committee of the Beritashvili Experimental Biomedicine Center.

\section{Surgical procedures}

Brain surgery was performed as described previously [19]. In short, under anesthesia with intramuscular administration of ketamine $(100 \mathrm{mg} / \mathrm{kg}$, "KharkovPharm", Ukraine), a 12-mm-long stainless steel guide cannula (Small Parts, Inc., USA) was stereotaxically implanted into the rostral part of ACC (area I) (AP: 2.70; L: +0.5; $\mathrm{H}: 2.5)$ according to the coordinates in the atlas of Paxinos and Watson (1997) [24] siting the tip $2 \mathrm{~mm}$ above the ACC. The guides were anchored to the cranium by dental cement. The guide cannula was plugged with a stainless steel stylet. Thereafter, the rats were handled every day for 3 days for 15 min to get familiar with the testing protocol and experimental environment. During this time, the stylet was removed and $14 \mathrm{~mm}$-long stainless steel microinjection cannula was inserted into the guide cannula to reach the ACC, but no drug was injected. This helped to habituate the rats to the injection procedure and to reduce artifacts arising from mechanical manipulation during the test days. Five days after surgery the microinjection cannula, attached to a 50- $\mu$ l Hamilton syringe (Hamilton, Inc., USA), was joined to the guide cannula, and the drug was introduced through it while the rat was gently restrained.

\section{Drugs}

Diclofenac (diclofenac sodium, $75 \mu \mathrm{g} / 0.5 \mu \mathrm{l}$, Hemofarm, Serbia), ketorolac (ketorolac tromethamine, $90 \mu \mathrm{g} / 0.5 \mu \mathrm{l}$, Grindex, Latvia) or xefocam (lornoxicam, $12 \mu \mathrm{g} / 0.5 \mu \mathrm{l}$, Nycomed, Austria) were injected through the microinjection cannula as we used in previous works $[19,20]$. The guide cannula was then plugged with a stainless steel stylet. Isotonic saline was injected in the same volume (0.5 $\mu \mathrm{l}$, GalichPharm, Ukraine) and manner in a separate group of rats for controls. In the second set of experiments a non-selective opioid receptor antagonist naloxone $(0.2 \mu \mathrm{g} / 0.5 \mu \mathrm{l}$, Polfa S.A., Poland) was injected through the microinjection cannula [19]. Solutions were microinjected in about $10-12 \mathrm{~s}$.

\section{Behavioral testing}

Twenty minutes post microinjection of NSAIDs, i.e. 10min before the peak of the drugs' effect is normally 
reached, animals were tested for antinociception using the tail-flick (TF) and hot plate (HP) tests. For the TF test, the distal part of the tail was stimulated with a light beam and the latency measured until the tail was reflexively flicked away from the beam (IITC \#33, IITC Life Science, Inc., Woodland Hills, CA, USA). For the HP test, the rat was placed on a $55{ }^{\circ} \mathrm{C}$ hot plate and the latency to the first hindpaw licking or jumping was measured (IITC \#39). The cut-off time was $20 \mathrm{~s}$ for both TF and HP latencies. Each rat was tested with both TF and HP in the same session. A similar procedure was followed for the repeated microinjection of diclofenac, ketorolac, xefocam or saline for four consecutive days. In special control experiments, saline microinjections into the ACC was followed by a non-selective opioid receptor antagonist naloxone $(0.5 \mu \mathrm{l}$, Polfa S.A., Poland) and tested for TF and HP latencies.

In the second set of experiments, pretreatment of rats with a non-selective opioid receptor antagonist naloxone in the ACC was followed by TF and HP tests. $10 \mathrm{~min}$ after they were treated with NSAIDs in the same dose as in the first set of experiments and were then tested again. Different animal groups were used for the first and second sets of experiments.

\section{Histology}

At the end of each set of experiments, the microinjection sites were marked with $2 \mu$ of saturated solution of Direct Blue-1 (Sigma-Aldrich) and the animal was euthanized with pentobarbital. After fixation by immersion in $10 \%$ formalin, the brain was sectioned and counterstained with Cresyl Violet. The microinjection sites were histologically verified and plotted according to Paxinos and Watson (1997) stereotaxic atlas coordinates [24]. Representative microinjection sites are shown in Fig. 1.

\section{Statistical analysis}

All data are presented as mean \pm S.E.M. One-way analysis of variance (ANOVA) or repeated measures of analysis of variance (rMANOVA) with post-hoc TukeyKramer or Dunnett multiple comparison tests were used for statistical comparisons between treated and saline groups, and treated and naloxone groups, respectively. The Kolmogorov-Smirnov test was applied to verify normality. The statistical software utilized was InStat 3.05 (GraphPad Software, USA). Differences between means of vehicle control and treated groups, and naloxone and treated groups of rats were acknowledged as statistically significant if $P<0.05$.

\section{Results}

Tolerance to antinociceptive effects of NSAIDs

In the first set of experiments we found that microinjection of NSAIDs into the ACC produced antinociception

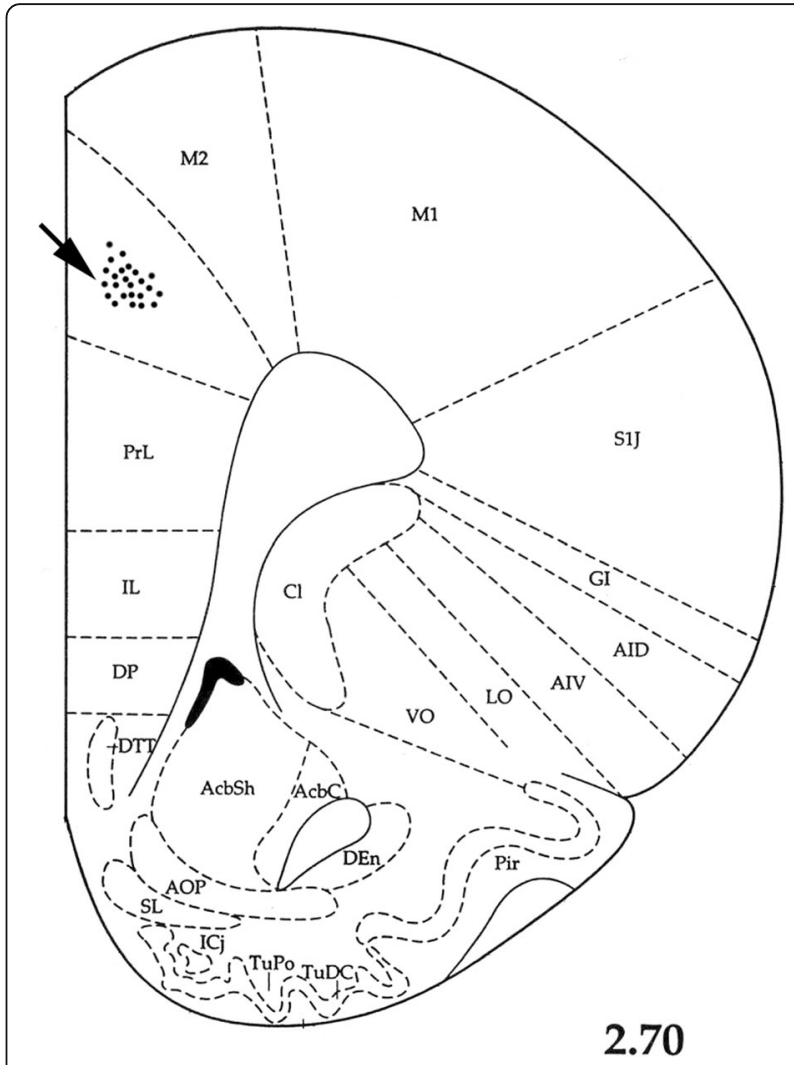

Fig. 1 A serial coronal section of the rat brain showing placement of microinjections unilaterally in the ACC (the black arrow). The number to the below of the section represents millimeters relative to bregma, adapted from the Paxinos and Watson (1997) stereotaxic atlas [24]

as detected by a latency increase in TF and HP compared to the baseline control of intact rats and a control group with saline microinjected into the same site as well. The rMANOVA revealed that the TF latency significantly increased for clodifen $[\mathrm{F}(9,20)=24.222, P<$ $0.0001]$, ketorolac $[\mathrm{F}(9,20)=71.399, P<0.0001]$, and xefocam $[F(9,20)=101.13, P<0.0001]$, respectively, but not for saline group $[F(9,20)=0.4148, P=0.7955$, not significant]. The TF latency differences between NSAIDs treated groups and the saline group by Dunnett test were significant in the first experimental day for diclofenac $(t=3.608, P<0.01)$, ketorolac $(t=3.424, P<0.01)$, and xefocam $(t=3.741, P<0.01)$, respectively (Fig. 2a).

We found similar significant differences in the HP latencies for diclofenac $[F(9,20)=29.045, P<0.0001]$, for ketorolac $[\mathrm{F}(9,20)=55.307, P<0.0001]$, and for xefocam $[F(9,20)=90.93, P<0.0001]$, respectively, but not for saline control $[\mathrm{F}(9,20)=1.299, P=0.3123$, not significant]. The HP latency differences between NSAIDs treated groups and the control group by Dunnett test were significant in the first experimental day for diclofenac $(t=2.687, P<0.05)$ and xefocam $(t=2.728, P<0.05)$, but not for ketorolac $(t=1.846, P>0.05)$ (Fig. 2b). 

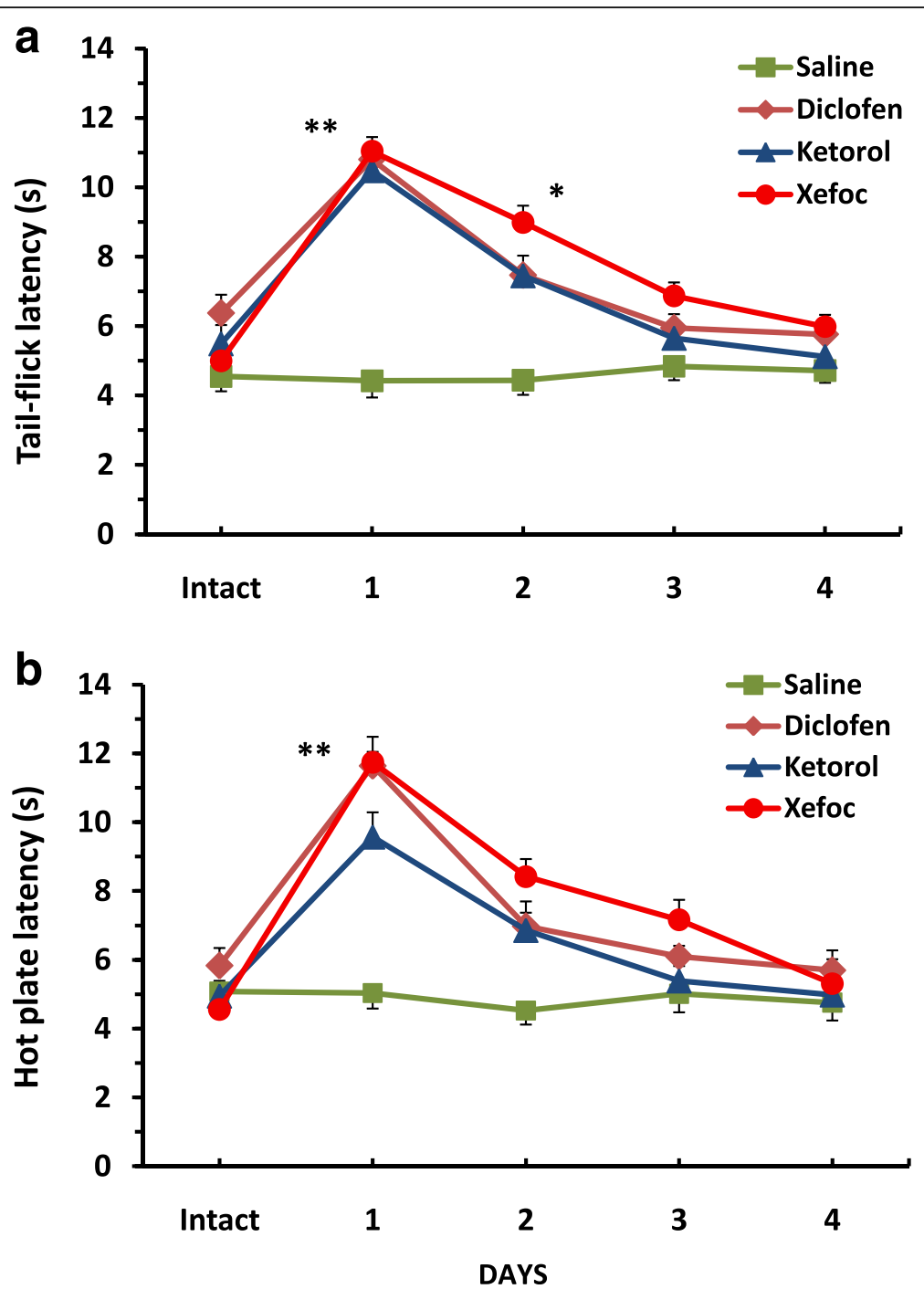

Fig. 2 Microinjections of NSAIDs into the ACC for 4 consecutive days result in a progressive decrease in TF (a) and HP (b) latencies as compared to vehicle saline control. ${ }^{*}-P<0.05,{ }^{*}{ }^{*}-P<0.01$

Subsequent NSAIDs microinjections caused progressively less antinociception, so by day 4 there was no effect, similar to saline microinjections for both the TF and the HP tests, i.e. induced tolerance. By the second experimental day the TF latency differences between NSAIDs treated groups and the saline group were significant only for xefocam $(t=3.066, P<0.05)$. There were not significant differences between NSAIDs treated groups and the control in the HP test for the second experimental day (Fig. 2).

\section{Pretreatment with naloxone prevents NSAIDs-induced antinociception}

In the second set of experiments, we tested if pretreatment with a non-selective opioid receptor antagonist naloxone prevents antinociception induced by NSAID microinjected into the ACC. Pretreatment with naloxone completely prevented the analgesic effects of diclofenac, ketorolac, and xefocam in the TF test. The differences between NSAIDs injected and naloxone injected groups are not significant [ANOVA: $\mathrm{F}(3,32)=1.419, P=0.2552$, not significant] (Fig. 3). The same results are in the HP test for diclofenac, ketorolac, and xefocam, respectively [ANOVA: $\mathrm{F}(3,32)=1.829, P=0.1618$, not significant] (Fig. 4).

Special control testing with naloxone microinjections into the ACC followed by saline statistically did not change the latency to respond in the TF [ANOVA: $\mathrm{F}(2,15)=1.301, P=0.3012$, not significant] (Fig. 3a), and HP [ANOVA: $F(2,15)=0.2939, P=0.2939$, not significant] tests, respectively (Fig. 4a).

\section{Discussion}

The data reported in this study demonstrate that microinjection of commonly used NSAIDs, diclofenac, ketorolac 

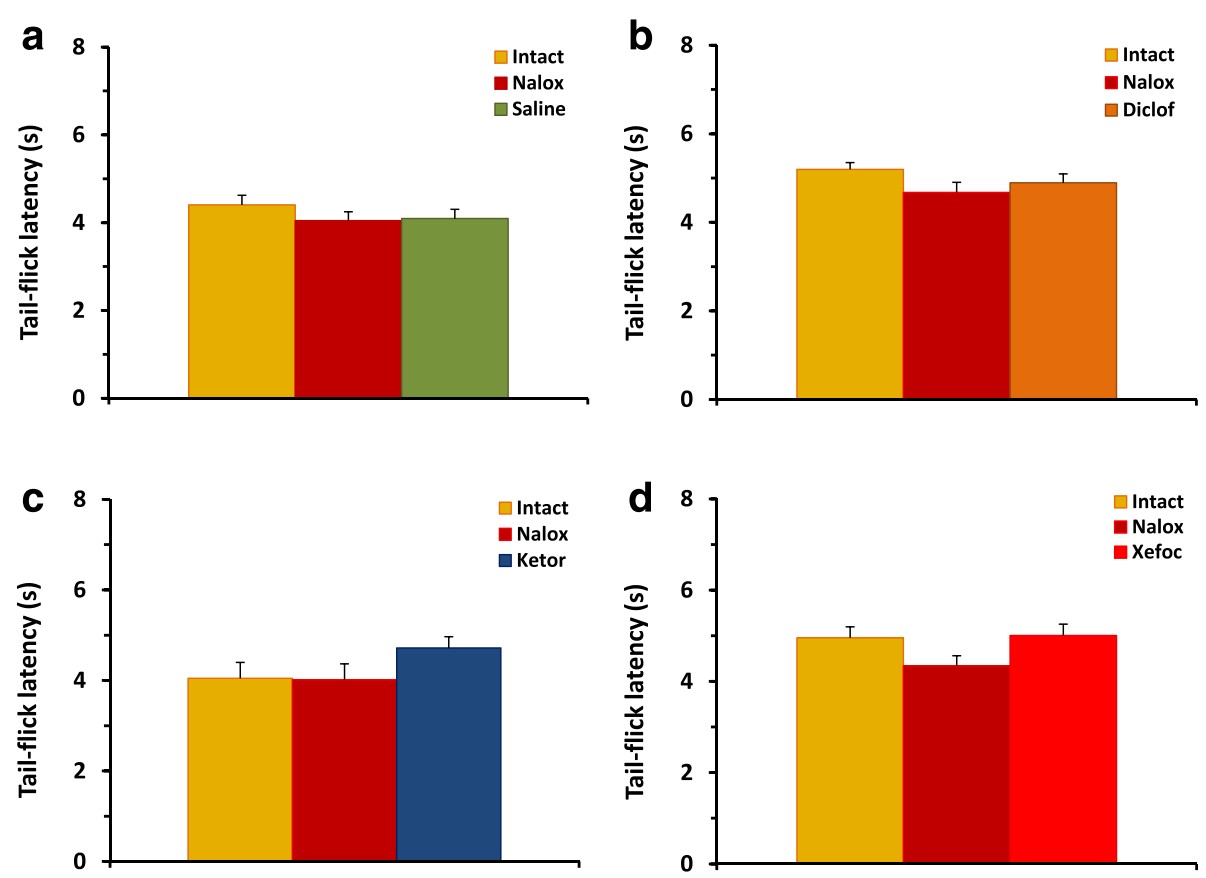

Fig. 3 Pretreatment with naloxone before microinjections of NSAIDs into the ACC results in prevention of NSAID-induced antinociception in TF latency for diclofenac $(\mathbf{b})$, ketorolac $(\mathbf{c})$, and xefocam (d), respectively. Control experiments of pretreatment with naloxone before microinjection of saline into the ACC do not significantly change TF latency (a)
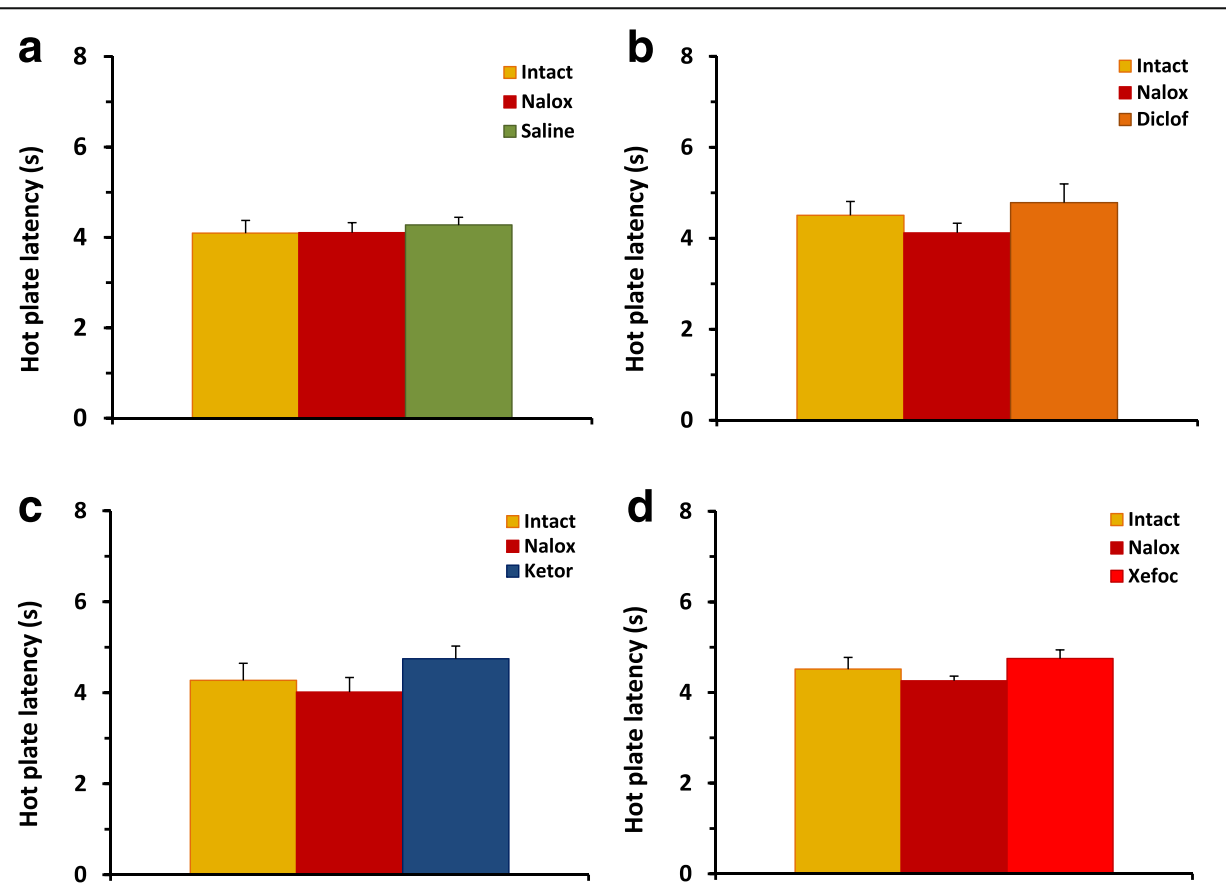

Fig. 4 Pretreatment with naloxone before microinjections of NSAIDs into the ACC results in prevention of NSAID-induced antinociception in HP latency for diclofenac (b), ketorolac (c), and xefocam (d), respectively. Control experiments of pretreatment with naloxone before microinjection of saline into the ACC do not significantly change HP latency (a) 
and xefocam into the rostral part of ACC induces antinociception. These findings are in resemblance with the results of our and other colleagues' previous investigations in an acute pain model with TF and HP tests, and in which metamizol, xefocam, ketorolac or lysineacetylsalicylate were given systemically or microinjected into the PAG [10, 11, 16-18, 25], into the CeA [13, 21], and the NRM $[13,20,26]$. In the other investigation, responses of spinal dorsal horn wide-dynamic range neurons of rats to mechanical noxious stimulation of a hindpaw were strongly inhibited by intravenous metamizol [27].

More importantly, repeated administrations of these NSAIDs into the ACC over a period of 4 days resulted in a progressive decrease in antinociceptive effectiveness, i.e. development of tolerance, reminiscent of that induced by opiates $[10,12,13,18,28]$. The present data confirm our previous results in which development of tolerance was observed to the analgesic effects of diclofenac, ketorolac and xefocam microinjected into the $\mathrm{DH}$ of rats. After injection of each drug, a progressive decrease in TF and HP latency (i.e., tolerance) was noticed over the 4-day period $[19,26]$.

The mechanism producing tolerance to NSAIDs can be due to the participation of endogenous opioids $[13,22,29,30]$. Here we clearly showed that pretreatment of a non-selective opioid receptor antagonist naloxone significantly diminishes NSAIDs-induced antinociception. These findings confirm our previous evidence where pretreatment with naloxone prevented antinociceptive effects of metamizol, ketorolac and xefocam in juvenile and adult rats. Moreover, in morphine-tolerant juvenile and adult rats we revealed effects of cross-tolerance to metamizol, ketorolac and xefocam [18]. As stated above, NSAIDs antinociception in the $\mathrm{DH}$ was reduced by pre- and posttreatment with naloxone $[19,26]$. Just recently we showed that systemic pretreatment with naloxone completely prevented the analgesic effects of NSAIDs (diclofenac, ketorolac and xefocam, i.p.) in thermal paw withdrawal (Hargreaves) test and mechanical paw withdrawal (von Frey) test in a chronic inflammatory pain model, the formalin test [31].

These and the present data also confirm previous results that anti-nociception induced by systemic metamizol involves endogenous opioids that can be blocked by naloxone at the levels of the PAG, the NRM and the spinal dorsal horn [32], as well as other findings that endogenous opioids are involved in the potentiation of analgesia observed with a combination of morphine plus dipyrone [33]. These data suggest a role for endogenous opioidergic descending pain control circuits. The latter consists of the brainstem pain modulatory network with critical links in the PAG as well as the rostral ventro-medial medulla [22, 29, 30, 34].

\section{Conclusions}

Similar to previous studies, we have demonstrated that administration of diclofenac, ketorolac and xefocam, widely used non-opioid, NSAID analgesics, into the rostral part of the ACC, induces antinociception in rats. When administered repeatedly, tolerance developed to the antinociceptive effects of these drugs. The present findings support the concept that the development of tolerance to the antinociceptive effects of NSAIDs is mediated via an endogenous opioid system possibly involving descending pain modulatory systems.

\section{Abbreviations}

ACC: The anterior cingulate cortex; ANOVA: The analysis of variance; CeA: The central nucleus of amygdala; DH: The dorsal hippocampus; HP: Hot plate; i.p.: Intraperitoneal; NRM: The nucleus raphe magnus; NSAIDs: Non-steroidal anti-inflammatory drugs; PAG: The periaqueductal grey matter; S.E.M.: Standard error of the mean; TF: The tail-flick

\section{Acknowledgments}

The authors greatly appreciate Prof. Earl Carstens for his English corrections to the manuscript.

\section{Funding}

This research did not receive any specific grant from funding agencies in the public, commercial, or not-for-profit sectors.

\section{Availability of data and materials}

The datasets analyzed during the current study are available from the corresponding author on reasonable request.

\section{Authors' contributions}

$N T, N P, I N$ and GG equally contributed to data collection. MN carried out histological control. EA and MGT designed conception, finally analyzed the data and drafted the manuscript. All authors read, contributed to and approved the final manuscript.

\section{Consent for publication}

Not applicable. This manuscript contains no personal data.

\section{Competing interests}

The authors declare that they have no competing interests.

\section{Publisher's Note}

Springer Nature remains neutral with regard to jurisdictional claims in published maps and institutional affiliations.

Received: 27 April 2017 Accepted: 21 December 2017

Published online: 06 January 2018

\section{References}

1. Seymour B, Dolan RJ. Emotion, motivation, and pain. In: McMahon SB, et al., editors. Wall and Melzacks' textbook of pain. 6th ed. Philadelphia: Elsevier; 2013. p. 248-55

2. Craig KD. Emotions and psychobiology. In: McMahon SB, et al., editors. Wall and Mellzack's textbook of pain. 5th ed. London: Elsevier; 2006. p. 231-40.

3. McKenna JE, Melzack R. Analgesia produced microinjection in dentate gyrus. Pain. 1992;49:105-12.

4. Tasker RAR, Choiniere M, Libman SM, Melzack R. Analgesia produced by injection of lidocaine into the lateral hypothalamus. Pain. 1987;31:237-48.

5. Vaccarino AL, Melzack R. Analgesia produced by injection of lidocaine into the anterior cingulum bundle of the rat. Pain. 1989;39:213-20. 
6. Corkin S, Hebben N. Subjective estimates of chronic pain before and after psychosurgery or treatment in a pain unit. Pain. 1981;1981(Suppl. 1):S150.

7. Casey KL. Concepts of pain mechanisms: the contributions of functional imaging of the human brain. Prog Brain Res. 2000;129:277-87.

8. Johansen JP, Fields HL, Manning BH. The affective component of pain in rodents: direct evidence for a contribution of the anterior cingulate cortex. PNAS. 2001;98:8077-82

9. Ito T, Tanaka-Mizuno S, Iwashita N, Tooyama I, Shiino A, Miura K, et al. Proton magnetic resonance spectroscopy assessment of metabolite status of the anterior cingulate cortex in chronic pain patients and healthy controls. J Pain Res. 2017;10:287-93.

10. Pernia-Andrade AJ, Tortorici $\mathrm{V}$, Vanegas $\mathrm{H}$. Induction of opioid tolerance by lysine-acetylsalicylate in rats. Pain. 2004;111:191-200.

11. Tortorici V, Aponte $Y$, Acevedo $H$, Nogueira L, Vanegas $H$. Tolerance to nonopioid analgesics in PAG involves unresponsiveness of medullary painmodulating neurons in male rats. Eur J Neurosci. 2009;29:1188-96.

12. Tortorici $V$, Nogueira $L$, Aponte $Y$, Vanegas $H$. Involvement of cholecystokinin in the opioid tolerance induced by dipyrone (metamizol) microinjections into the periaqueductal gray matter of rats. Pain. 2004;112:113-20.

13. Tsagareli MG, Tsiklauri N. Behavior study of 'non-opioid tolerance'. New York: Nova Biomedical; 2012

14. Tsagareli MG, Tsiklauri N, Nozadze I, Gurtskaia G. Tolerance effects of NSAIDs microinjected into central amygdala, periaqueductal grey, and nucleus raphe: possible cellular mechanism. Neural Regen Res. 2012;7:1029-39.

15. Vanegas $H$, Tortorici $V$. Opioidergic effects of non-opioid analgesics on the central nervous system. Cell Mol Neurobiol. 2002;22:655-61.

16. Vanegas $\mathrm{H}$, Tortorici $\mathrm{V}$. The periaqueductal gray as critical site for antinociception and tolerance induced by non-steroidal anti-inflammatory drugs. In: Maione S, Di Marzo V, editors. Neurotransmission in the Antinociceptive descending pathway. Kerala: Research Signpost; 2007. p. 69-80.

17. Tsiklauri N, Gurtskaia G, Tsagareli MG. Study of non-opioid analgesic tolerance in young and adult rats. Georgian Med News. 2008;5:40-4

18. Tsiklauri N, Viatchenko-Karpinsky V, Voitenko N, Tsagareli MG. Non-opioid tolerance in juvenile and adult rats. Eur J Pharmacol. 2010;629:68-72.

19. Gurtskaia G, Tsiklauri N, Nozadze I, Nebieridze M, Tsagareli MG. Antinociceptive tolerance to NSAIDs microinjected into dorsal hippocampus. BMC Pharmacol Toxicol. 2014;15:10. https://doi.org/10.1186/ 2050-6511-15-10.

20. Tsagareli MG, Nozadze I, Tsiklauri N, Gurtskaia G. Tolerance to non-opioid analgesics is opioid sensitive in the nucleus raphe magnus. Front Neurosci. 2011;5:92. https://doi.org/10.3389/fnins.2011.00092.

21. Tsagareli MG, Tsiklauri N, Gurtskaia G, Nozadze I, Abzianidze E. The central nucleus of amygdala is involved in tolerance to the antinociceptive effect of NSAIDs. Health. 2010;2:64-8.

22. Vanegas H, Vazquez E, Tortorici V. NSAIDs, opioids, cannabinoids and the control of pain by the central nervous system. Pharmaceuticals. 2010;3:1335-47.

23. Zimmermann M. Ethical guidelines for investigations of experimental pain in conscious animals. Pain. 1983;16:109-10.

24. Paxinos G, Watson C. The rat brain in Stereotaxic coordinates. San Diego: Academic Press; 1997.

25. Tortorici $\mathrm{V}$, Vanegas $\mathrm{H}$. Opioid tolerance induced by metamizol (dipyrone) microinjections into the periaqueductal gray of rats. Eur J Neurosci. 2000;12: 4074-80.

26. Tsiklauri N, Nozadze I, Firkulashvili N, Gurtskaia G, Nebieridze M, Abzianidze E, Tsagareli MG. Cellular mechanisms of antinociceptive tolerance to nonsteroidal anti-inflammatory drugs. In: systemic, cellular and molecular mechanisms of physiological functions and their disorders, Chapter 26. New York: Nova Biomedical; 2016. p. 339-62.

27. Telleria-Diaz A, Schmidt M, Kreusch S, Neubert AK, Schache F, Vazquez E, et al. Spinal antinociceptive effects of cyclooxygenase inhibition during inflammation: involvement of prostaglandins and endocannabinoids. Pain. 2010;148:26-35.

28. Tortorici V, Nogueira L, Salas R, Vanegas H. Involvement of local cholecystokinin in the tolerance induced by morphine microinjections into the periaqueductal gray of rats. Pain. 2003;102:9-16.

29. Heinricher MM, Ingram SL. The brainstem and nociceptive modulation. In: Basbaum Al, Bushnell MC, editors. Science of pain. San Diego: Elsevier; 2009. p. 593-626.

30. Heinricher MM, Fields HL. Central nervous system mechanisms of pain modulation. In: McMahon SB, et al., editors. Wall \& Melzack's textbook of pain. 6th ed. Philadelphia: Elsevier; 2013. p. 129-42.
31. Tsiklauri N, Nozadze I, Gurtskaia G, Tsagareli MG. Antinociceptive tolerance to NSAIDs in the rat formalin test is mediated by the opioid mechanism. Pharmacol Reports. 2017;69:168-75.

32. Vazquez E, Hernandez N, Escobar W, Vanegas H. Antinociception induced by intravenous dipyrone (metamizol) upon dorsal horn neurons: involvement of endogenous opioids at the periaqueductal gray matter, the nucleus raphe magnus, and the spinal cord in rats. Brain Res. 2005;1048:211-7.

33. Hernández-Delgadillo GP, Cruz SL. Endogenous opioids are involved in morphine and dipyrone analgesic potentiation in the tail flick test in rats. Eur J Pharmacol. 2006;546:54-9.

34. Vazquez E, Escobar W, Ramirez C, Vanegas H. A non-opioid analgesic acts upon the PAG-RVM axis to reverse inflammatory hyperalgesia. Eur J Neurosci. 2007;25:471-9.

\section{Submit your next manuscript to BioMed Central and we will help you at every step:}

- We accept pre-submission inquiries

- Our selector tool helps you to find the most relevant journal

- We provide round the clock customer support

- Convenient online submission

- Thorough peer review

- Inclusion in PubMed and all major indexing services

- Maximum visibility for your research

Submit your manuscript at www.biomedcentral.com/submit
) Biomed Central 